\title{
O método pilates na melhora do equilíbrio da população idosa brasileira: uma
}

\section{revisão de literatura}

\author{
The pilates method in improving the balance of the elderly brazilian population: a literature review \\ El método pilates para mejorar el equilibrio de la población brasileña mayor: una revisión de la
}

literatura

Recebido: 06/09/2021 | Revisado: 08/09/2021 | Aceito: 08/09/2021 | Publicado: 11/09/2021

\author{
Isabella Freitas e Silva \\ ORCID: https://orcid.org/0000-0001-9248-656X \\ Centro Universitário da Amazônia, Brasil \\ E-mail: bellafreitasesilva18@gmail.com \\ Roberta Francisca Mota Albuquerque \\ ORCID: https://orcid.org/0000-0001-6823-5517 \\ Centro Universitário da Amazônia, Brasil \\ E-mail: robertinhaamota09@gmail.com \\ Indiara de Alencar \\ ORCID: https://orcid.org/0000-0003-3204-3278 \\ Universidade do Estado do Pará, Brasil \\ E-mail: indiara.alencarstm@gmail.com
}

\begin{abstract}
Resumo
O Pilates está se tornando um dos tratamentos mais indicados e buscados pela população idosa brasileira além de colaborar para a saúde física e mental correlacionando com as atividades de vida diária dos idosos. Com isso, o estudo buscou informações que colaboram na melhora da flexibilidade, tônus muscular, equilíbrio postural, propriocepção e assim minimizando outras patologias, como doenças cardiorrespiratórias e musculoesqueléticas, além de colaborar na diminuição das internações. De acordo com este estudo podemos observar resultados positivos no aumento da flexibilidade e na funcionalidade, assim melhorando o equilíbrio postural dos idosos, consequentemente minimizando os riscos de quedas. Desta maneira buscou-se o método como alternativa de intervenção terapêutica para contribuir na qualidade de vida dos idosos, com isso o estudo pretendeu maneira positiva para construção de novos projetos acadêmicos e na divulgação ao público alvo.
\end{abstract}

Palavras-chave: Fisioterapia; Equilíbrio; Idosos; Pilates.

\begin{abstract}
Pilates is becoming one of the most indicated and sought after treatments by the elderly Brazilian population, in addition to contributing to physical and mental health, correlating with the elderly's daily activities. Thus, the study sought information that helps to improve flexibility, muscle tone, postural balance, proprioception and thus minimize other pathologies, such as cardiorespiratory and musculoskeletal diseases, in addition to helping to reduce hospitalizations. According to this study, we can observe positive results in increasing flexibility and functionality, thus improving the postural balance of the elderly, consequently minimizing the risk of falls. In this way, the method was sought as an alternative therapeutic intervention to contribute to the quality of life of the elderly, with this the study intended a positive way to build new academic projects and dissemination to the target audience.
\end{abstract}

Keywords: Physiotherapy; Balance; Elderly; Pilates.

\section{Resumen}

Pilates se está convirtiendo en uno de los tratamientos más recomendados y buscados por la población anciana brasileña, además de contribuir a la salud física y mental, correlacionándose con las actividades diarias de las personas mayores. Así, el estudio buscó información que ayude a mejorar la flexibilidad, el tono muscular, el equilibrio postural, la propiocepción y así minimizar otras patologías, como las enfermedades cardiorrespiratorias y musculoesqueléticas, además de ayudar a reducir las hospitalizaciones. Según este estudio, podemos observar resultados positivos en el aumento de la flexibilidad y funcionalidad, mejorando así el equilibrio postural de las personas mayores, minimizando en consecuencia el riesgo de caídas. Así, se buscó el método como una intervención terapéutica alternativa para contribuir a la calidad de vida de las personas mayores, con esto el estudio pretendía construir positivamente nuevos proyectos académicos y difundirlo al público objetivo.

Palabras clave: Fisioterapia; Equilibrio; Anciano; Pilates. 


\section{Introdução}

De acordo com a Organização Mundial da Saúde (2005), o envelhecimento é um evento que pode ser destacado por um processo natural de toda a humanidade, ocorrendo ao longo da vida. Com o avanço da idade sucede doenças e distúrbios ostemioarticulares correlacionados ao envelhecimento, destaca-se as patologias degenerativas. A diminuição da funcionalidade é uma das consequências que afetam a população idosa, assim diminuindo a capacidade musculoesquelética de conduzir suas atividades da vida diária. Dentre algumas modificações que decorre por conta do envelhecimento, destaca-se as correlacionadas ao equilíbrio corporal, ocorrendo uma diminuição do tempo de reação nas instabilidades corporais estáticas e dinâmicas (Mesquita, et al., 2015).

A queda pode ser caracterizada pela alteração repentina e não premeditada da posição, levando o paciente a um nível inferior (Paraizo, \& Iwamoto, 2018). De acordo com Ensured et al., (2007) por conta das suas decorrências no bem-estar dos idosos, considera-se a queda como uma sequência de eventos, apresentando relação com a fragilidade, perda do equilíbrio, influenciando na dependência do indivíduo, apresentando as sequelas como fraturas e até a morte. Com isso, as quedas são vistas como problemas para a saúde pública e o cotidiano dos idosos (Gelbard, et al., 2014). Cerca de 25\% da população acima dos 65 anos sofrem por quedas em cada ano, destacando a fratura de fêmur como alto risco de dependência do idoso (Obrist, et al., 2016). O receio de cair resulta no aumento da perda da independência, na capacidade de realizar atividades da sua rotina, com isso patologias oriundas do sedentarismo e assim, gerando diminuição do equilíbrio postural e da mobilidade (Lopes, et al., 2009).

Diversos fatores influenciam na estabilidade, entre os fatores intrínsecos estão a instabilidade corporal, fraqueza musculares, doenças reumatológicas que afetam a parte motora, o próprio envelhecimento atinge a fisiologia do corpo e as alterações da marcha. Já os fatores extrínsecos associam-se com o ambiente, por exemplo, piso escorregadio, tapetes pela residência, objetos no chão, ausência de barras de apoio, móveis instáveis e iluminação inadequada (Ambrose, et al., 2013). Destacam-se os fatores extrínsecos como um dos principais fatores de risco ambientais, de acordo com os estudos representa até $50 \%$ das quedas entre os idosos. São fatores ligados onde o idoso transita, podemos destacar: tapetes, brinquedos pela área de circulação, escada sem apoio, iluminação inadequada, móveis inadequados, banheiro sem a barra e o piso escorregadio (Ambrose AF, Paul G \& Hausdorff JM, 2013). Com isso, os estudos relacionam a perca de equilíbrio postural, fraqueza muscular e distúrbios da marcha com os riscos ambientais e a presença de quedas (Rubenstein, 2006).

Nesse sentido, a fisioterapia tem um papel importante, na prevenção de quedas em idosos incluindo orientações, assistência na realização das atividades da vida diária, incluindo alongamentos, treino de marcha e equilíbrio, fortalecimento muscular e a manutenção da funcionalidade, promovendo a redução das limitações e incapacidades e colaborando com a independência desses idosos (Aveiro; Driusso \& Santos, 2013).

Por conta do crescimento da população idosa torna-se um assunto mundial, e, no Brasil, por conta dessa curva de desenvolvimento é de suma importância estudarmos recursos que melhorem o equilíbrio, um deles muito citados é o Pilates que está cada vez mais sendo visto pela população idosa brasileira (Carvalho, 2003 \& Garcia, 2008).

O método Pilates foi elaborado pelo alemão Joseph Hubertus Pilates (1880-1967), por conta das sequelas oriundas da Primeira Guerra Mundial o qual desenvolveu atividades para ganho de força muscular. Em 1980 a técnica do Pilates foi reconhecida no mundo inteiro e ganhou popularidade na reabilitação (Anderson, Spector, 2005; Muscolino \& Cipriani, 2004).

Segundo Figueiredo et al., (2018) considera-se o método Pilates bastante disseminado no Brasil, sendo um dos métodos realizados pelos fisioterapeutas no tratamento em diversas disfunções. De acordo com Barker et al., (2015) os princípios do Método Pilates são: controle, respiração, fluidez, concentração, precisão e centralização. No meio dos benefícios que o método traz que são o ganho de força muscular e resistência, melhora do equilíbrio, propriocepção, na flexibilidade, 
coordenação e conscientização corporal (Cruz-Díaz, et al., 2015). Segundo Bullo et al., (2015) o recurso contribui para a melhora da qualidade de vida, para os idosos estes benefícios são diversos e abrangem desde físico até os aspectos sociais:

[...] aumento da capacidade aeróbia; aumento na ventilação voluntária; melhora na flexibilidade; melhora na resistência muscular localizada; aumento do conteúdo de minerais ósseos; diminuição da resistência vascular; melhor tolerância à glicose; redução da concentração de lipídios; melhora do estado de ânimo, aumento da vitalidade e melhora significativa da qualidade de vida (Rocha, 2002 apud Curi, 2009, p. 22-23).

A fisioterapia através Método Pilates na população idosa brasileira, mostra-se eficaz quanto ao nível do tratamento de patologias ou alterações como osteoporose, diminuição da força muscular, alterações na postura e no equilíbrio (Gandolfi, et al., 2020). Assim, o objetivo dessa revisão de literatura é investigar os benefícios do Método Pilates na melhora do equilíbrio dos idosos.

\section{Metodologia}

A construção do presente estudo fundamentou-se em uma revisão bibliográfica de caráter descritivo, e estratégia qualitativa através do método hipotético dedutivo.

Para conduzir a elaboração dessa revisão determinamos como a questão problema: "De que forma a aplicação do Método Pilates influencia na melhora do equilíbrio da população idosa brasileira?"

Para elaboração do presente estudo buscou-se reunir artigos que se apresenta importância no campo científico sobre o tema proposto, foi realizado por meio de cinco itens, que são: definição de uma pergunta norteadora, seleção as bases de dados, filtragem os artigos, avaliação os estudos, construção os resultados e discussões. A pesquisa foi executada nas seguintes bases eletrônicas: Google Acadêmico, Physiotherapy Evidence Database (PEDro) e Scientific Electronic Library Online (SciELO), utilizando os descritores: fisioterapia, equilíbrio, idosos e pilates.

A análise foi feita a partir da leitura criteriosa dos artigos selecionados. Com o total de 15 estudos, onde foi verificado o método Pilates como forma de tratamento no público idoso brasileiro, sendo possível analisar o Método Pilates na melhora do equilíbrio postural.

Os critérios de inclusão foram trabalhos que tenham sido publicados entre os anos 2015 à 2021, dissertações, monografias, teses, estudo de caso, artigos que abordassem a temática sobre os benefícios do Pilates nos idosos, publicações na íntegra e de acesso livre, abordando a importância da fisioterapia com o público alvo e estudos publicados na língua portuguesa.

Como critérios de exclusão foram artigos não disponibilizados na íntegra, trabalhos duplicados, publicações anteriores ao ano de 2015, artigos que não tem coerência com o tema proposto, trabalhos não concluídos e de revisão bibliográfica, artigos pagos e artigos da língua estrangeira.

\section{Resultados e Discussão}

No Quadro 1 mostra-se 8 estudos realizados só em mulheres, 6 estudos realizados em ambos os sexos e 1 estudo realizado em homens, submetidos ao Método Pilates. 
Quadro 1 - Resultado dos estudos publicados nos anos de 2015 à 2021.

\begin{tabular}{|c|c|c|c|c|c|c|}
\hline Título & Autor & Amostra & Sexo & Idade & $\begin{array}{c}\text { Técnicas } \\
\text { Utilizadas }\end{array}$ & Resultados \\
\hline $\begin{array}{l}\text { Influência do método } \\
\text { pilates no equilíbrio de } \\
\text { idosos e seu Impacto } \\
\text { sobre o risco de quedas e } \\
\text { qualidade de vida: ensaio } \\
\text { Clínico controlado e } \\
\text { randomizado. }\end{array}$ & $\begin{array}{c}\text { ARAÚJO, S.S, } \\
2021 .\end{array}$ & $\begin{array}{c}7 \text { idosas do grupo } \\
\text { controle e } 7 \\
\text { idosas grupo } \\
\text { pilates. }\end{array}$ & Feminino & $\begin{array}{l}\text { A partir de } \\
60 \text { anos }\end{array}$ & $\begin{array}{l}\text { Método Pilates } \\
\text { no solo e cartilha } \\
\text { de prevenção. }\end{array}$ & $\begin{array}{l}\text { Após o protocolo de } \\
\text { exercícios de } \\
\text { fortalecimento e os } \\
\text { alongamentos, obtiveram } \\
\text { melhora do equilíbrio, } \\
\text { redução do risco de quedas } \\
\text { e qualidade de vida. }\end{array}$ \\
\hline $\begin{array}{l}\text { Análise comparativa de } \\
\text { Pilates e treinamento } \\
\text { resistido na aptidão física } \\
\text { de idosas. }\end{array}$ & $\begin{array}{l}\text { PUCCI, } \\
\text { G.C.M.F, et al, } \\
2021 .\end{array}$ & $\begin{array}{l}41 \text { idosas } \\
\text { distribuídas em } 3 \\
\text { grupos (grupo } \\
\text { pilates, grupo } \\
\text { treinamento } \\
\text { resistido e grupo } \\
\text { de atividades } \\
\text { lúdicas e } \\
\text { cognitivas). }\end{array}$ & Feminino & $\begin{array}{l}\text { A partir de } \\
60 \text { anos }\end{array}$ & $\begin{array}{l}\text { Método pilates e } \\
\text { exercícios } \\
\text { resistidos. }\end{array}$ & $\begin{array}{l}\text { Houve melhora em todas } \\
\text { as variáveis analisadas do } \\
\text { grupo pilates e } \\
\text { treinamento resistido, após } \\
24 \text { semanas de tratamento. }\end{array}$ \\
\hline $\begin{array}{l}\text { Qualidade de vida de } \\
\text { homens idosos } \\
\text { praticantes de pilates na } \\
\text { cidade de Pirapora -MG. }\end{array}$ & $\begin{array}{l}\text { CAMPOS, R.S, } \\
\text { et al, } 2021 .\end{array}$ & $\begin{array}{c}9 \text { idosos } \\
\text { praticantes. }\end{array}$ & Masculino & $\begin{array}{l}\text { A partir de } \\
60 \text { anos }\end{array}$ & $\begin{array}{l}\text { Método Pilates e } \\
\text { um questionário } \\
\text { sobre o estilo de } \\
\text { vida (QVS-80). }\end{array}$ & $\begin{array}{l}\text { Após aplicação de testes e } \\
\text { dos exercícios, observa-se } \\
\text { a melhora em todos os } \\
\text { aspectos dos idosos, tanto } \\
\text { físico e mental. }\end{array}$ \\
\hline $\begin{array}{l}\text { Análise do equilíbrio } \\
\text { postural em idosos } \\
\text { praticantes e não } \\
\text { praticantes de Pilates. }\end{array}$ & $\begin{array}{l}\text { CRUZ, } \\
\text { L.G.R.D, et al, } \\
2020 .\end{array}$ & 34 idosos. & $\begin{array}{l}\text { Ambo os } \\
\text { sexos }\end{array}$ & $\begin{array}{l}\text { A partir de } \\
68 \text { anos }\end{array}$ & $\begin{array}{l}\text { Mat Pilates } \\
\text { incluindo } \\
\text { exercícios no } \\
\text { solo e sem } \\
\text { equipamentos } \\
\text { específicos e no } \\
\text { aparelho } \\
\text { (reformador, } \\
\text { cadillac, unidade } \\
\text { de parede. } \\
\text { cadeira } \\
\text { combinada) }\end{array}$ & $\begin{array}{l}\text { Conclui-se que a prática } \\
\text { regular do Pilates } \\
\text { promoveu ganho de força } \\
\text { muscular, flexibilidade, } \\
\text { hipertrofia, controle } \\
\text { corporal e outros } \\
\text { componentes do equilíbrio } \\
\text { postural, como sistema } \\
\text { vestibular, visual e } \\
\text { proprioceptivo. }\end{array}$ \\
\hline $\begin{array}{l}\text { Efeitos do Método } \\
\text { Pilates no equilíbrio, na } \\
\text { força muscular e } \\
\text { flexibilidade em idosas. }\end{array}$ & $\begin{array}{l}\text { SANTOS, } \\
\text { B.F.D, et al, } \\
2020 \text {. }\end{array}$ & $\begin{array}{l}19 \text { idosas } \\
\text { saudáveis. }\end{array}$ & Feminino & $\begin{array}{l}\text { Acima de } \\
60 \text { anos }\end{array}$ & $\begin{array}{c}\text { Método do } \\
\text { Pilates no solo. }\end{array}$ & $\begin{array}{l}\text { Após } 10 \text { sessões de } \\
\text { fisioterapia, obteve } \\
\text { melhora na força } \\
\text { muscular, equilíbrio e na } \\
\text { flexibilidade. }\end{array}$ \\
\hline $\begin{array}{l}\text { A influência do método } \\
\text { Pilates no equilíbrio e } \\
\text { qualidade de vida do } \\
\text { idoso. }\end{array}$ & $\begin{array}{l}\text { DORATHY, } \\
\text { O.K; GUÉRIOS, } \\
\text { L, } 2019 .\end{array}$ & $\begin{array}{l}20 \text { idosos } \\
\text { saudáveis. }\end{array}$ & $\begin{array}{l}\text { Ambo os } \\
\text { sexos }\end{array}$ & $\begin{array}{c}\text { Entre } 65 \text { e } \\
86 \text { anos }\end{array}$ & $\begin{array}{l}\text { Exercícios do } \\
\text { Método Pilates } \\
\text { no solo. }\end{array}$ & $\begin{array}{c}\text { Após } 20 \text { sessões, os idosos } \\
\text { obtiveram melhora } \\
\text { significativa no equilíbrio } \\
\text { e na capacidade funcional. }\end{array}$ \\
\hline $\begin{array}{l}\text { Efeitos de } 16 \text { semanas do } \\
\text { método pilates mat sobre } \\
\text { o equilíbrio, marcha, } \\
\text { mobilidade funcional e } \\
\text { força de idosas, 60-79 } \\
\text { anos. }\end{array}$ & $\begin{array}{l}\text { NASCIMENTO, } \\
\text { M.D.M, et al, } \\
2019 .\end{array}$ & 48 idosas. & Feminino & $\begin{array}{c}60 \text { a } 79 \\
\text { anos }\end{array}$ & $\begin{array}{l}\text { Aulas do método } \\
\text { Pilates Mate ( } 2 \\
\text { dias por semana, } \\
60 \text { minutos). }\end{array}$ & $\begin{array}{l}\text { Demonstraram que após } \\
\text { aplicabilidade, foi efetivo } \\
\text { nos níveis de flexibilidade, } \\
\text { equilíbrio estático e } \\
\text { dinâmico, bem como a } \\
\text { mobilidade funcional. }\end{array}$ \\
\hline $\begin{array}{c}\text { Contribuições da prática } \\
\text { de pilates na aptidão } \\
\text { física e na força de } \\
\text { preensão manual de } \\
\text { idosos. }\end{array}$ & $\begin{array}{l}\text { PACHECO, } \\
\text { L.D.A, et al, } \\
2019 .\end{array}$ & 11 idosos. & $\begin{array}{l}\text { Ambo os } \\
\text { sexos }\end{array}$ & $\begin{array}{l}\text { Acima de } \\
68 \text { anos }\end{array}$ & $\begin{array}{c}\text { Realizaram } 34 \\
\text { sessões de } \\
\text { exercícios do } \\
\text { Pilates de Solo. }\end{array}$ & $\begin{array}{l}\text { Obteve melhora da força, } \\
\text { flexibilidade dos membros } \\
\text { inferiores, } \\
\text { agilidade/equilíbrio } \\
\text { dinâmico e resistência } \\
\text { aeróbia. }\end{array}$ \\
\hline $\begin{array}{c}\text { Intervenção pelo método } \\
\text { Pilates no solo: } \\
\text { influência sobre o }\end{array}$ & $\begin{array}{c}\text { MELLO, J.S.S, } \\
\text { et al, } 2019 .\end{array}$ & 10 idosos. & $\begin{array}{l}\text { Ambo os } \\
\text { sexos }\end{array}$ & $\begin{array}{l}\text { A partir de } \\
60 \text { anos }\end{array}$ & $\begin{array}{c}\text { Método Pilates } \\
\text { solo, realizaram } \\
3 \text { meses de } \\
\end{array}$ & $\begin{array}{c}\text { De acordo com o estudo } \\
\text { obteve melhora } \\
\text { parcialmente no }\end{array}$ \\
\hline
\end{tabular}




\begin{tabular}{|c|c|c|c|c|c|c|}
\hline $\begin{array}{l}\text { desempenho motor, } \\
\text { funcional e cognitivo de } \\
\text { idosos. }\end{array}$ & & & & & tratamento. & $\begin{array}{l}\text { desempenho funcional, } \\
\text { porém no cognitivo e } \\
\text { motor obtiveram } \\
\text { resultados satisfatórios. }\end{array}$ \\
\hline $\begin{array}{l}\text { O tempo de prática e a } \\
\text { prática de outro exercício } \\
\text { físico influenciam na } \\
\text { aptidão física de idosos } \\
\text { praticantes do método } \\
\text { pilates? }\end{array}$ & $\begin{array}{c}\text { OLIVEIRA, } \\
\text { D.V, et al, } 2019 .\end{array}$ & 60 idosos. & $\begin{array}{l}\text { Ambo os } \\
\text { sexos }\end{array}$ & $\begin{array}{l}\text { Entre } 60 \mathrm{e} \\
80 \text { anos }\end{array}$ & $\begin{array}{l}\text { Método Pilates e } \\
\text { testes de } \\
\text { flexibilidade e } \\
\text { força. }\end{array}$ & $\begin{array}{l}\text { Através do estudo, foi } \\
\text { analisado a importância do } \\
\text { maior tempo da prática do } \\
\text { Pilates, o quanto contribui } \\
\text { na flexibilidade dos idosos } \\
\text { estudados. }\end{array}$ \\
\hline $\begin{array}{l}\text { Equilíbrio postural e } \\
\text { risco de quedas de idosas } \\
\text { praticantes do método } \\
\text { pilates e idosas } \\
\text { sedentárias. }\end{array}$ & $\begin{array}{l}\text { KAROLCZAK, } \\
\text { A.P, et al, } 2017 .\end{array}$ & 30 idosas. & Feminino & $\begin{array}{c}\text { Entre } 60 \mathrm{e} \\
80 \text { anos }\end{array}$ & Método Pilates. & $\begin{array}{l}\text { Que a prática do método } \\
\text { Pilates por idosas preserva } \\
\text { o equilíbrio dinâmico e } \\
\text { estático e a coordenação } \\
\text { motora, e diminui o risco } \\
\text { de quedas. }\end{array}$ \\
\hline $\begin{array}{l}\text { Avaliação na } \\
\text { funcionalidade de uma } \\
\text { idosa praticante do } \\
\text { método pilates. }\end{array}$ & $\begin{array}{l}\text { GUEDES, } \\
\text { M.J.D.N, et al, } \\
2017 .\end{array}$ & 1 idosa. & Feminino & 77 anos & $\begin{array}{l}\text { Exercícios foi } \\
\text { aplicado com } \\
\text { base no Mat } \\
\text { Pilates. }\end{array}$ & $\begin{array}{l}\text { Trouxe melhoras no ganho } \\
\text { de flexibilidade, equilíbrio } \\
\text { dinâmico e estático, } \\
\text { melhora no alinhamento } \\
\text { postural e da percepção } \\
\text { corporal. }\end{array}$ \\
\hline $\begin{array}{l}\text { Efeito do Método Pilates } \\
\text { na postura e no } \\
\text { equilíbrio. dinâmico de } \\
\text { idosas }\end{array}$ & $\begin{array}{l}\text { CAMARGO, } \\
\text { M.B, et al, } 2016 \text {. }\end{array}$ & 18 idosas. & Feminino & $\begin{array}{c}\text { Entre } 60 \text { e } \\
75 \text { anos }\end{array}$ & $\begin{array}{c}\text { Foram } \\
\text { submetidas a } 30 \\
\text { sessões de Mat } \\
\text { Pilates, } 2 \text { vezes } \\
\text { por semana. }\end{array}$ & $\begin{array}{c}\text { De acordo com as } \\
\text { particularidades das } \\
\text { idosas, obtiveram melhora } \\
\text { no equilíbrio e na postura. }\end{array}$ \\
\hline $\begin{array}{l}\text { Autonomia funcional dos } \\
\text { idosos praticantes do } \\
\text { método pilates }\end{array}$ & $\begin{array}{l}\text { CRISPIM } \\
\text { D.C.S, et al, } \\
\quad 2016 .\end{array}$ & $\begin{array}{c}50 \text { idosos } \\
\text { praticantes e } \\
\text { sedentários }\end{array}$ & $\begin{array}{c}\text { Ambos os } \\
\text { sexos }\end{array}$ & $\begin{array}{c}\text { Entre } 60 \mathrm{e} \\
74 \text { anos }\end{array}$ & Método Pilates & $\begin{array}{l}\text { Colabora significamente } \\
\text { para a evolução da } \\
\text { autonomia } \\
\text { funcional nos idosos. }\end{array}$ \\
\hline $\begin{array}{l}\text { Análise do efeito do } \\
\text { método Pilates no perfil } \\
\text { postural de idosas }\end{array}$ & $\begin{array}{l}\text { MUNIZ, J.N, et } \\
\text { al, } 2015 .\end{array}$ & 20 idosas & Feminino & $\begin{array}{c}\text { Entre } 62 \text { e } \\
74 \text { anos }\end{array}$ & $\begin{array}{l}\text { Aplicação do } \\
\text { método Pilates, } \\
\text { um seguimento } \\
\text { de } 20 \text { sessões, } \\
\text { duas por semana }\end{array}$ & $\begin{array}{c}\text { A realização dos } \\
\text { exercícios do método } \\
\text { Pilates promoveu um } \\
\text { efeito positivo na postura } \\
\text { das idosas. }\end{array}$ \\
\hline
\end{tabular}

Fonte: Silva, Albuquerque e Alencar (2021).

No presente estudo foi possível analisar a efetividade do Método Pilates como intervenção para melhoria do equilíbrio em idosos, além de intervir como alternativa positiva no ganho de força, flexibilidade e funcionalidade como foi observado ao longo da pesquisa. Para se obter resultados positivos foi observado que em $78 \%$ dos estudos analisados a prática do Pilates foi efetuada em uma frequência de 2 a 3 vezes na semana, entre 40 a 60 minutos com o número de sessões superior a 20. Porém, em apenas $11 \%$ dos resultados foi demonstrado que o Método Pilates não influenciou significativamente no equilíbrio dinâmico de idosos. $\mathrm{O}$ autor desta pesquisa atribuiu o resultado negativo à falha na autoconsciência de imagem corporal e aptidão motora por parte dos voluntários, fator que, segundo Camargo et al., 2016, influencia negativamente na precisão dos exercícios, diminuindo sua efetividade devido à dificuldade no entendimento do procedimento dos exercícios. Apesar disso, o pesquisador ressaltou que com uma constância e orientação mais adequadas poderiam se obter melhores resultados.

Concomitante a importância da frequência dos exercícios, a eficiência de se inserir as técnicas respiratórias no tratamento é válida, uma vez que, Kovalek e Guérios (2019) abordaram em sua pesquisa sobre os princípios do método Pilates que são: respiração, concentração, centro, controle, precisão e fluxo dos movimentos, os quais devem ser adaptados de acordo com as peculiaridades de cada indivíduo, acompanhando os exercícios solo do método para trabalho de força, equilíbrio e 
flexibilidade. Somando-se a isso, Santos et al., 2020, atribuiu a melhoria do quadro de seus voluntários nos quesitos equilíbrio, força abdominal e de extensores de troco, e flexibilidade, à técnica de respiração lateral associada à expiração do volume de reserva respiratório durante os exercícios.

Além disso, vale ressaltar a importância da estabilização postural para melhoria do equilíbrio em idosos por meio do Pilates observada durante a pesquisa. Isso é possível através do Pilates ao trabalhar os músculos abdominais e paravertebrais, que são músculos posturais, considerados o centro do corpo segundo Joseph Pilates (Endleman \& Critchley, 2008). Em 100\% dos estudos foi comprovada o aumento da força nesses músculos, somente uma pesquisa apresentou um aumento discreto, o qual o autor atribuiu à má postura adotada de flexão leve de tronco ao contrair os músculos do abdômen durante os exercícios, não permitindo que seja alcançada a amplitude final do movimento, além do período de intervenção reduzido, que deveria ser maior pois a postura dos idosos apresenta alterações estruturadas tornando-as difíceis de serem corrigidas em um curto período (Camargo, et al., 2016).

Ademais, Muniz et al., 2015 demonstrou por meio do instrumento de avaliação postural SAPO, uma melhora significativa na postura de uma idosa após intervenção com Pilates. No mesmo estudo também foi observado, por meio da escala de Tinetti, um aumento de 16 pontos no score de avaliação do equilíbrio, comprovando a eficácia do método associando equilíbrio e postura.

Portanto, considerando que é perceptível que idosos ativos praticantes de qualquer atividade física podem prolongar sua autonomia funcional e qualidade de vida quando comparados à idosos sedentários (Crispim, et al., 2016), pode-se concluir que o método Pilates é uma alternativa de atividade a ser considerada, uma vez que este método é eficiente nos ganhos em equilíbrio e postura, essenciais para funcionalidade, por meio de exercícios de baixa intensidade, os quais não agridem as articulações e ajudam a proporcionar independência e autonomia, sendo necessário a prática dessa atividade com uma frequência maior e por um período mais longo a fim de aumentar seus efeitos positivos (Muniz, et al., 2015).

Tal efetividade pode ser comprovada pelos relatos de praticantes que aumentaram seu vigor e disposição para realizar suas atividades diárias, permitindo notar que o método também contribui para melhora de dores e autoestima, além de convívio social saudável (Kovalek \& Guérios, 2019).

Vale salientar que foi observada uma predominância de amostras do sexo feminino, sendo necessária realização de mais estudos com voluntários masculinos para se ter mais precisão nos resultados, considerando as diferenças fisiológicas e anatômicas entre os sexos. Também foi notado a necessidade de mais pesquisas com grupos maiores de voluntários em pesquisas randomizadas para se obter resultados mais precisos. Por conseguinte, pode-se concluir que o Pilates é um método essencial a ser aplicado em idosos.

Compreendemos que o Pilates engloba um conjunto de funcionalidade, assim promovendo a melhora do equilíbrio, postura, ajuda no fortalecimento muscular e assim diminuindo os riscos de quedas entre os idosos, colaborando significamente para autonomia para o mesmo e assim melhorando a qualidade de vida, porém de acordo com o estudo de (Mello, et al., 2019) após o tratamento que ocorreu em 12 semanas de Pilates solo, podemos analisar que os 10 idosos estudados obtiveram melhora parcialmente na funcionalidade e já no aspecto cognitivo, flexibilidade e força não se teve resultados positivos, pois de acordo com os autores a prática deveria ter sido regulamente, faça-se a importância de mais estudos nesta linha de raciocínio, para melhor entendimento na junção da cognição e o Pilates.

Além disso Oliveira et al., (2019) estudaram sobre o tempo de prática e a prática de outro exercício físico influenciam na aptidão física de idosos praticantes do método pilates, observa-se que os idosos que praticam o método não se tem uma diferença relevante com os idosos que praticam exercícios. Todavia, a quantidade de tempo da prática do Pilates, ao que tudo indica que, favorecem melhores efeitos acerca da flexibilidade dos membros superiores nos idosos estudados pelos autores, pois os mesmos passaram por testes de flexibilidade e de força. 
Estudos recentes, como de Campos et al., 2021 que foi estudado com 9 idosos sobre a qualidade de vida de homens idosos praticantes de pilates em uma cidade de Minas Gerais. Após o questionário (QVS-80), observou-se que de acordo com as respostas os idosos que participaram regularmente das atividades físicas por volta de dois anos, porém todos os idosos informaram sobre alguma lesão ou desconforto que tiveram ao longo dessa prática de exercícios, no entanto sem intervenções médicas, os entrevistados mostraram resultados satisfatórios no quesito: integração social (família, amigos e sociedade), sobre a percepção da qualidade de vida foi avaliada como ótima na grande parte dos participantes, alguns fatores como mobilidade, flexibilidade, equilíbrio e força apresentaram respostas satisfatórias, autor até correlaciona a prática do pilates com esses resultados positivos, sabemos que o método é uma possibilidade de tratamento que contribui para o sistema musculoesquelético dos idosos, assim prevenindo doenças crônicas oriundas do processo do envelhecimento. Dessa forma, para os futuros estudos, conseguiremos mostrar que o Pilates não contribui só na qualidade de vida e sim em todo os sistemas do corpo humano.

No estudo de Pucci e seus colaboradores, (2021) mostra-se um estudo comparativo sobre o Pilates e o treinamento resistido em idosos, o qual os autores dividiram as idosas em três grupos: grupo pilates, grupo treinamento resistido e grupo de atividades lúdicas e cognitivas, foram submetidas em um tratamento que cada grupo realizava o exercício duas vezes por semana, duravam em média 60 minutos de atividade, durante 24 semanas, foi realizado o teste antes da intervenção e após intervenção. Foi observado que obtiveram melhora em vários fatores como: equilíbrio, resistência aeróbica, flexibilidade e força muscular em ambos os grupos, no entanto, o estudo quer mostrar o quanto o Pilates e o exercícios resistidos colaboraram para a melhora da aptidão física.

Já o estudo sobre a influência do método pilates no equilíbrio de idosos e seu impacto sobre o risco de quedas e qualidade de vida foi desenvolvido e implementado um programa de exercícios de Pilates solo, as idosas foram divididas em dois grupos: grupo pilates e o grupo cartilha orientando sobre os riscos de quedas, o tratamento durou três meses, foi observado que houve melhora no equilíbrio postural das idosas e os fatores analisados como: dor, capacidade funcional, aspectos sociais, emocionais e aspectos físicos. De acordo com os achados do estudo, podemos analisar que o Pilates colabora na redução dos riscos de quedas e assim melhorando a qualidade de vida, vale ressaltar que temos que analisar de acordo com cada grupo, pois um grupo foi só orientado com a cartilha, (Araújo, 2021).

Segundo o estudo de Cruz et al., 2020 podemos analisar o quanto o pilates contribui em todos os aspectos do corpo humano principalmente para os praticantes, assim podemos observar que melhora na força muscular, flexibilidade e equilíbrio postural. Portanto, o pilates colaborou para a melhora principalmente do equilíbrio dinâmico, assim reduz o número de quedas no público idoso e automaticamente melhorando a qualidade de vida. Podemos compreender que o pilates vai além da melhora do equilíbrio.

\section{Conclusão}

De modo geral, podemos observar que as pesquisas voltadas para a utilização do Método Pilates na melhora do equilíbrio dos idosos brasileiros nos últimos anos, tornam-se cada vez mais alvo de estudo. Além disso, o recurso mostra-se efetivo no alinhamento postural, conscientização corporal, mobilidade, tais benefícios contribuem para diminuição de quedas.

Através dos resultados obtidos nesse estudo podemos finalizar que o Método Pilates é uma forma de tratamento, que fornece benefícios na funcionalidade do público idoso, visto que contribui no treinamento físico, diminui as alterações posturais decorrentes do envelhecimento, melhora na flexibilidade, melhora no equilíbrio, propriocepção, força muscular, amenizando as dores oriundas de patologias, melhora os aspectos sociais e emocionais dos idosos, ajuda na concentração, coordenação motora, assim contribui em todos os sistemas do corpo humano, proporcionando a melhora da qualidade de vida. 
Sendo que a qualidade de vida é uma das condições básicas para manter sua saúde em boas condições e podemos associar ao Método Pilates pois, podemos observar através desse estudo literário que o método é seguro e prazeroso de realizar com o público idoso e assim contribuindo para a sua independência das suas atividades da vida diária. Recomenda-se novos estudos que contém amostra e que proporcionem a melhora do equilíbrio corporal na população idosa.

\section{Agradecimentos}

Agradecemos nossa orientadora Indiara de Alencar que sempre esteve presente, incentivou, orientou e apoiou nosso estudo. Somos eternamente gratas pela sua dedicação.

\section{Referências}

Araújo, J. (2021). Influência do método pilates no equilíbrio de idosos e seu impacto sobre o risco de quedas e qualidade de vida: ensaio clínico controlado e randomizado.

Ambrose, A. F., Paul, G. \& Hausdorff, J. M. (2013). Risk factors for falls among older adults: a review of the literature. Maturitas.

Anderson, B. D. \& Spector, A. (2005). Introduction to Pilates-Based Rehabilitation. Balanced Body.

Aveiro, M. C., Driusso, P. \& Santos, J. G. (2013). Effects of a physical therapy program on quality of life among community-dwelling elderly women: randomized-controlled trial. Revista Fisioterapia em Movimento, Curitiba. 26(3), 503-13.

Bullo, V., Bergamin, M., Gobbo, S., Sieverdes, J.C., Zaccaria, M., Neunhaeuserer, D., \& Ermolao, A. (2015). The effects of Pilates exercise training on physical fitness and wellbeing in the elderly: A systematic review for future exercise prescription. Preventive Medicine, n. 75, p. 1-11.

Brouwer, B. J., Walker, C., Rydahl, S. J. \& Culham, E. G. (2003). Reducing fear of falling in seniors through education and activity programs: a randomized trial. J Am Geriatr Soc 51(6), 829-34.

Camargo, M. B, et al. (2016). Efeito do Método Pilates na postura e no equilíbrio dinâmico de idosas. Fisioter Bras; (3):236-43.

Campos, R. S., Nascimento, U. C., Veloso, M. V. \& Martins, L. P. O. (2021). Qualidade de vida de homens idosos praticantes de pilates na cidade de Pirapora - MG. Bionorte. https://doi.org/10.47822/2526-6349.2021v10n1p127.

Carvalho, J. A. M. \& Garcia, R. A. (2003). O envelhecimento da população brasileira: um enfoque demográfico. Rio de Janeiro: Cad. Saúde pública, 19, 726732.

Crispim, D. C. S, et al. (2016). Autonomia funcional dos idosos praticantes do método pilates. R. Interd. 9(3), 12-17.

Cruz-Diaz, D, et al. (2015). Effects of a six week Pilates intervention on balance and fear of falling in women aged over 65 with chronic low back pain: A randomized controlled trial. Maturita, (82), 371-376.

Dorathy, O. K. \& Guérios, L. (2019). A influência do método Pilates no equilíbrio e qualidade de vida do idoso. Fisioterapia Brasil, (4):S15-S21.

Endleman I. \& Critchley D. J. (2008). Atividade transverso abdominal e oblíqua interna durante os exercícios de pilates: medição com varredura de ultrassom. Arch Phys Med Rehabil.

Ensured, K. E, et al. (2007). Frailty and risk of falls, fracture, and mortality in older women: the study of osteoporotic fractures. J Gerontol Ser A Biol Sci Med Sci.

Figueiredo, T, et al. (2018). Intervenção do método pilates em idosos no brasil: uma revisão sistemática. Rev. Aten. Saúde, 16(57), 87-97.

Graziely, L., Cruz, R., Effgen, E. B. P., Liposcki, D. B., Almeida, M. G., Camargos, J. C. V. \& Rinaldi, N. M. (2020). Análise do equilíbrio postural em idosos praticantes e não praticantes de Pilates. Rev Bras Fisiol Exerc. https://doi.org/ 10.33233/rbfe.v19i3.365.

Gandolfi, N, et al. (2020). The influence of the Pilates method on quality of life and bone remodelling in older women: a controlled study. Quality of Life Research.

Gelbard, R, et al. (2014). Falls in the elderly: a modern look at an old problem. Am J Surg.

Guedes, M. J. D. N, et al. (2017). Avaliação na funcionalidade de uma idosa praticante do método pilates. Interscientia, vol 5, n.1.

Hartholt, K. A, et al. (2011). Increase in fall-related hospitalizations in the United States, 2001-2008. J Trauma.

Karolczak, A. P, et al. (2017). Equilíbrio postural e risco de quedas de idosas praticantes do método pilates e idosas sedentárias, FisiSenectus. Unochapecó.

Lopes, et al. (2009). Prevalence of fear of falling among a population of older adults and its correlation with mobility, dynamic balance, risk and history of falls. Revista Brasileira de Fisioterapia, 13(3), 223-229. 
Research, Society and Development, v. 10, n. 11, e595101120299, 2021

(CC BY 4.0) | ISSN 2525-3409 | DOI: http://dx.doi.org/10.33448/rsd-v10i11.20299

Mesquita, L. S. A, et al. (2015). Effects of two exercise protocols on postural balance of elderly women: a randomized controlled trial. BMC Geriatrics, $15(61), 1-9$

Mello, J. S. S., Oliveira, D. V. D., Pivetta., N. R. S., Bertolini, S. M. M. G. (2019). Intervenção pelo método Pilates no solo: influência sobre o desempenho motor, funcional e cognitivo de idosos. Arch. Health. Sci. 26(1):15-18.

Muniz, J. N, et al. (2015). Análise do efeito do método Pilates no perfil postural de idosas. ConScientiae Saúde, 14(3), 410-416.

Nascimento, M. D. M, et al. (2019). Efeitos de 16 semanas do método pilates mat sobre o equilíbrio, marcha, mobilidade funcional e força de idosas, 60-79 anos. Revista Saúde (Sta. Maria).

Oliveira, D. V. D, et al. (2019). O tempo de prática e a prática de outro exercício físico influenciam na aptidão física de idosos praticantes do método pilates? Rev. Aten. Saúde, 17(62), 31-39.

Organização Mundial de Saúde (OMS), (2005). O papel da atividade física no Envelhecimento saudável.

Pacheco, L. D. A, et al. (2019). Contribuições da prática de pilates na aptidão física e na força de preensão manual de idosos. Arq. Cienc. Saúde UNIPAR, Umuarama, 23(3), 189-

Paraizo, V. M. C, et al. (2018). Protocolo de prevenção de quedas. Américas serviços médicos.

Pucci, G. C. M. F, et al. (2021). Análise comparativa de Pilates e treinamento resistido na aptidão física de idosas. Retos, número 41.

Rocha, P. E. C. (2002). Medidas e Avaliação em ciências do Esporte. (5a ed.), Sprint.

Rubenstein, L. Z. (2006). Falls in older people: Epidemiology, risk factors and strategies for prevention. Age and Ageing, 5(2), 37-41.

Saari, P, et al. (2007). Fall-related injuries among initially 75- and 80-year old people during a 10-year follow-up. Arch Gerontol Geriatr.

Santos, B. F. D, et al. (2020). Efeitos do Método Pilates no equilíbrio, na força muscular e flexibilidade em idosas. PAJAR, 8. 